\title{
HOMOGENIZATION OF MESOSCOPIC THEORIES: EFFECTIVE PROPERTIES OF MODEL MEMBRANES
}

\author{
R. Lam ${ }^{1}$, D. G. Vlachos ${ }^{2 *}$, M. A. Katsoulakis ${ }^{3}$ \\ ${ }^{1}$ Department of Chemical Engineering, University of Massachusetts, Amherst, MA 01003-3110 \\ ${ }^{2}$ Department of Chemical Engineering and Center for Catalytic Science and Technology (CCST), \\ University of Delaware, Newark, DE 19716-3110 \\ ${ }^{3}$ Department of Mathematics and Statistics, University of Massachusetts, Amherst, MA 01003- \\ 3110
}

\begin{abstract}
A new mathematical framework is introduced for modeling diffusion in nanoporous materials or on surfaces exhibiting heterogeneity in properties over large length scales while retaining molecular scale information typically captured by molecular simulations only. This framework entails first the use of newly developed mesoscopic equations derived rigorously from underlying master equations by coarse-graining statistical mechanics techniques. Homogenization techniques are then employed to derive the leading-order effective mesoscopic models that are subsequently solved by spectral methods. These solutions are also compared to direct numerical simulations for selected two-dimensional model membranes with defects, when attractive adsorbate-adsorbate interactions affect particle diffusion. It has been found that not only the density but also the dispersion of defects significantly alters the macroscopic behavior in terms of fluxes and concentration patterns, especially when phase transitions can occur. It is also shown that homogenization techniques could potentially offer a promising alternative to direct numerical simulations, when complex, large-scale heterogeneities are present. Implications for various applications, including heterogeneous catalysts, materials growth, and separations using membranes, are also discussed.

* Corresponding author.
\end{abstract}




\section{INTRODUCTION}

The introduction of Monte Carlo (MC) techniques 1 in conjunction with the rapidly increasing computational power have revolutionized our understanding about the mesoscopic structure, thermodynamic, and transport properties of a large spectrum of problems for which molecular interactions are important. Examples include bulk liquids, solids, surface reconstruction phenomena, protein folding, and crystallization ${ }^{2-6}$. Aside from equilibrium problems, MC methods, especially on a lattice, have been successfully employed for irreversible problems such as crystal growth and catalytic reactions 7-12. MC simulations solve directly an underlying master equation, and given sufficient information about transition probabilities, they provide its exact solution.

Despite the enormous progress achieved so far, MC simulations are computationally very intensive and limited to relatively small time and length scales. The issue of length scales is not restrictive when only microscopic inhomogeneities are present as a result, for example, of intermolecular forces. These can typically be captured adequately via periodic boundary conditions and sufficiently large simulation boxes. However, there are three broad classes of problems, which are currently intractable by MC simulations due to macroscopic inhomogeneities. The first class includes systems that exhibit mesoscopic or macroscopic patterns as a result of self-regulation mechanisms. Examples include Turing patterns 13 and patterns resulting from the competition of microphase separation, driven by attractive interactions, and chemical reaction 14, 15. The second class encompasses forced systems that operate under an overall macroscopic gradient in one or more variables (e.g., concentration, temperature, etc.). Examples include transport through membranes, catalytic reactors, and chemical vapor deposition reactors. The last class involves systems that exhibit intrinsic heterogeneity in properties over relatively large scales than those amenable to MC simulations. Examples include composite materials, heterogeneous catalysts, and materials with mesoscopic defects. To our knowledge, problems in this last class in which intermolecular forces dictate microscopic mechanisms of transport and chemistry have not been previously addressed, and are therefore the focus of this paper.

A recently introduced mesoscopic approach 16-18, 15 based on coarse-graining of the underlying master equation provides a promising alternative to MC simulations when larger scales need to be considered. Such mesoscopic models can describe exactly the role of intermolecular forces in diffusion under certain conditions. Mesoscopic equations retain the nature of the microscopic dynamics they are based upon (e.g., Metropolis versus Arrhenius), can capture anisotropic interactions and stochastic effects (e.g., nucleation), and incorporate multiple additional processes such as desorption, reaction, and adsorption. Recent numerical simulations and theoretical results have demonstrated the excellent performance of this novel approach 17, 19, 20 , as briefly outlined in the next section. Being continuum equations, numerical solutions of mesoscopic equations can be easily and efficiently obtained for long time and large length scales, especially when spectral algorithms are employed ${ }^{14}$. Therefore, solutions of the aforementioned first two classes of spatially inhomogeneous systems can be systematically obtained.

Here we extend this mathematical mesoscopic framework to the third class of spatially inhomogeneous materials. In particular, an effective mesoscopic diffusion model, including strong interparticle interactions and material heterogeneity, is formulated. This new mathematical framework is applied to model membranes in two-dimensions. The organization of this paper is as follows. After briefly introducing the features of the mesoscopic equations, 
homogenization techniques are briefly reviewed and applied. The latter approach enables us to develop approximate solutions for heterogeneous systems in different scaling regimes. Effective permeation properties of model membranes are then computed under an overall concentration gradient using both direct numerical simulations and homogenization. Direct numerical simulations, using spectral methods, allow us to validate the homogenized solutions and to examine the effect of heterogeneity on concentration patterns and on the overall permeation behavior. Finally, conclusions are drawn.

\section{MESOSCOPIC MODELING}

The mesoscopic equations are coarse-grained limits of the master equations as the range of the interaction potential approaches infinity and are exact in this limit. Although mesoscopic equations can be derived for a variety of processes 16-18, 15, here we mainly focus on diffusion. For Metropolis and Arrhenius microscopic dynamics, the respective mesoscopic diffusion equations are ${ }^{17}$ :

$$
\begin{aligned}
& \frac{\partial \mathrm{u}}{\partial \mathrm{t}}-\mathrm{d} \nabla \cdot\{[\nabla \mathrm{u}-\beta \mathrm{u}(1-\mathrm{u}) \nabla \mathrm{K} * \mathrm{u}]\}=0 \\
& \frac{\partial \mathrm{u}}{\partial \mathrm{t}}-\mathrm{D} \nabla \cdot\left\{\mathrm{e}^{-\beta \mathrm{K}^{*} \mathrm{u}}[\nabla \mathrm{u}-\beta \mathrm{u}(1-\mathrm{u}) \nabla \mathrm{K} * \mathrm{u}]\right\}=0,
\end{aligned}
$$

where $u$ is the coverage (dimensionless concentration), $D=d e^{-\beta U_{o}}$ is the diffusion coefficient, $d$ is the diffusion coefficient at high (infinite) temperature, $\mathrm{U}_{\mathrm{o}}$ is the energy associated with the binding to the host, $\mathrm{K}$ is the intermolecular potential of adsorbate-adsorbate interactions, $\mathrm{K} * \mathrm{u}=\int \mathrm{K}\left(\left|\mathrm{r}-\mathrm{r}^{\prime}\right|\right) \mathrm{u}\left(\mathrm{r}^{\prime}\right) \mathrm{dr} \mathrm{r}^{\prime}$ is the convolution and $\beta^{-1}=\mathrm{kT}$, with $\mathrm{k}$ being the Boltzmann constant and $\mathrm{T}$ being the temperature.

For many systems, the range of the interactions can typically be relatively short suggesting that mesoscopic models may have limited accuracy. However, it has been previously shown, via one- and two-dimensional simulations 17,20 , that the mesoscopic formalism can still yield accurate results even for relatively short-range interactions because these equations exhibit a Large Deviation Principle ${ }^{19}$. The latter principle suggests that the infinite potential length case results should be approached exponentially fast as the potential length increases. These conclusions appear independent of details of the potential (attractive or repulsive, piecewise constant or continuous, etc.) and microscopic mechanisms of diffusion ${ }^{17,20}$. In fact, as often happens with the global mean field theory for equilibrium problems, theoretical predictions and numerical simulations suggest that excellent agreement is attained with shorter potentials in higher dimensions.

As an example, Figure 1 compares results from 1D and 2D gradient continuous time MC (G-CTMC) simulations (solid lines) 17, 20 to those from the corresponding mesoscopic equation (dashed lines) for diffusion under an overall concentration gradient, for the geometry depicted in the inset of Figure 1a. Dirichlet boundary conditions of 1 and 0 in the concentration are imposed in the direction of transport and periodic boundary conditions are imposed in the transverse one in the 2D simulations. A piecewise constant potential of constant strength (attractive interactions) was used in these simulations. In particular, the potential was taken constant and equal to $\mathrm{K}=\mathrm{w} / \mathrm{N}_{\mathrm{p}}$, where $\mathrm{N}_{\mathrm{p}}$ is the number of neighbors within the range of the potential $1_{\mathrm{p}}$, and zero farther away. Arrhenius microscopic dynamics ${ }^{17}$ was employed in these simulations. The upper two concentration profiles in each panel are shifted on the vertical axis for clarity. 
Excellent agreement between the two approaches (MC and mesoscopic) is achieved even for relatively short-range interactions. Despite some deviations seen in concentration profiles for short potentials (e.g., $\mathrm{l}_{\mathrm{p}}=1$ ), the corresponding fluxes of the G-CTMC and mesoscopic models differ only up to a few percent ${ }^{20}$. Furthermore, comparable deviations between the $1 \mathrm{D}$ and $2 \mathrm{D}$ simulations are observed for potential lengths in 2D roughly equal to the square root of those in 1D. This dimensionality effect has been rigorously shown theoretically in ${ }^{19}$, and indicates that shorter potentials are needed in higher dimensions to reach the infinite range scale results.

In the next section, effective mesoscopic diffusion equations for heterogeneous systems will be derived using homogenization techniques. While the results are independent of boundary conditions, in later sections, the configuration depicted in the inset of Figure la will be used as a prototype example to compute effective properties. Physically, this configuration mimics permeation experiments through nanoporous films, such as those encountered in zeolite membranes. An example of the microstructure of a MFI zeolite supported film fabricated by the secondary growth technique, which was obtained recently using laser confocal microscopy ${ }^{21}$, is shown in Figure 2d. The bright areas indicate grain boundaries (defects) within the zeolite film. More defects are observed near the alumina substrate at the bottom of the film. As shown below, this asymmetry in microstructure is important regarding permeation properties, when intermolecular forces are important.

\section{HOMOGENIZATION}

Homogenization techniques are a set of formal mathematical procedures that allow for the inclusion of smaller-scale heterogeneities in a lager scale problem. The mathematical foundations can be found in $22-24$ and have been applied to a large variety of problems including composite materials modeling 25 , porous media 26-29, and climatic and geomechanic modeling 30. Their major advantage is that they can provide effective properties of a relatively large heterogeneous medium for which application of direct numerical simulations is too intensive.

In the problem of interest, they are several scales present as shown in Figure 2. These include the microscopic lattice constant (site-to site-jump distance), the interaction potential scale $l_{p}$ (panel $c$ ), the heterogeneity scale $l_{h}$ which represents the length scale capturing statistically the variations in the materials properties (panel b), and the macroscopic system scale $\mathrm{L}$ (panel a), making the problem intrinsically multiscale. The heterogeneity scale is taken to be much smaller than the macroscale, which is equivalent, here, to assuming that the heterogeneity is periodic, although it is possible to apply homogenization to randomly distributed systems ${ }^{31}$. In the numerical examples considered below, the macroscopic and heterogeneity scales have the units of the cutoff distance of the potential.

With this assumption, three different scaling regimes can still appear. The potential range (cutoff distance) can be smaller than, similar to, or larger than the heterogeneity scale. Although the methodology is very general, for clarity, the homogenization procedures will be presented here for one scaling regime only, the case when the potential range is much larger than the heterogeneity scale. This situation may arise when long range electrostatic interactions dominate. Let us define a macroscopic variable $\mathrm{x}, \mathrm{x}=\left(\mathrm{x}_{1}, \mathrm{x}_{2}\right)$ in two-dimensions, that describes the behavior at the scale $\mathrm{L}$ and similarly a microscopic variable $\mathrm{y}$ that describes the behavior at the small scale $1_{h}$, as depicted in Figure 2, so that the following relations hold:

$$
\varepsilon=\frac{l_{h}}{L}<<1 \text { and } y=\frac{x}{\varepsilon} .
$$


The heterogeneity of the material can manifest itself in the diffusion coefficient and/or in the interparticle interactions. Let $\mathrm{D}=\mathrm{D}(\mathrm{y})$ be periodic in the microscale $\mathrm{y}\left(\mathrm{l}_{\mathrm{h}}\right.$ periodic) and $\mathrm{K}=$ $\mathrm{K}(\mathrm{x}, \mathrm{y})$. Physically, for spherical symmetric potentials the interaction between two particles depends only on the distance between them and the nature of the medium around each particle. The distance between two particles can be greater or smaller than the characteristic length associated the heterogeneity scale. Therefore, $\mathrm{K}$ depends on both $\mathrm{x}$ and $\mathrm{y}$. The nature of the medium at the position of the particle is purely dependent on the microscale since the heterogeneity is taken to be microscopic. Thus we will consider a potential of the form:

$$
\mathrm{K}\left(\mathrm{x}_{\mathrm{A}}, \mathrm{x}_{\mathrm{B}}, \mathrm{y}_{\mathrm{A}}, \mathrm{y}_{\mathrm{B}}\right)=\mathrm{J}\left(\left|\mathrm{x}_{\mathrm{A}}-\mathrm{x}_{\mathrm{B}}\right|\right) \mathrm{a}\left(\mathrm{y}_{\mathrm{A}}\right) \mathrm{b}\left(\mathrm{y}_{\mathrm{B}}\right) \text {. }
$$

Here, $\mathrm{J}$ corresponds to the distance term and would represent the interaction potential in a homogeneous medium; $a$ and $b$ are the medium indicator terms. For simplicity in the homogenization procedure, the terms $a$ and $b$ are taken to be symmetric $(a=b)$ and their variation in amplitude is taken to be of the same order as the characteristic scale of the heterogeneity. Physically, this symmetry arises from the fact that a pair of particles A and B interacts the same way, whether viewed from A or B. In the more general case, where the amplitude of the variations in the potential caused by heterogeneities, is unrelated to the size of the heterogeneity, another small parameter could be introduced to characterize the variations of the potential. This would render the homogenization procedure a multiple parameter expansion. The terms $\mathrm{a}$ and $\mathrm{b}$ are taken to be weakly convergent, here, so that they can be expressed as:

$$
\begin{aligned}
& a=a_{0}+\varepsilon a_{1}+\varepsilon^{2} a_{2}+\ldots \\
& b=b_{0}+\varepsilon b_{1}+\varepsilon^{2} b_{2}+\ldots
\end{aligned}
$$

The concentration in the mesoscopic equation depends on both scales and is expanded as:

$$
u=u(x, y, t)=u_{0}+\varepsilon u_{1}+\varepsilon^{2} u_{2}+\ldots
$$

Using the above notation, as an example in the Metropolis case, we can recast Eq. 1 as follows:

$$
u_{t}(x, y)=\operatorname{div}\left[D(y)\left[\begin{array}{l}
\nabla u(x, y)- \\
\left.\beta u(x, y)(1-u(x, y)) \nabla\left(\int J\left(x-x^{\prime} \mid\right)\left(\frac{x}{\varepsilon}\right) b\left(\frac{x^{\prime}}{\varepsilon}\right) u\left(x^{\prime}, \frac{x^{\prime}}{\varepsilon}\right) d x^{\prime}\right)\right]
\end{array}\right] .\right.
$$

The first step in obtaining the homogenized effective equation is expanding (7) using (6), (5), and (4) to form cell boundary-value problems at each order of $\varepsilon$. The leading order problem is homogeneous and its solution is either independent of the microscopic scale or indeterminate. Next, linearity is invoked to express the higher order solutions in terms of the leading order one and construct an inhomogeneous canonical cell problem. From this inhomogeneous cell problem, the effective governing equation of the leading order can be expressed. In this example, the inhomogeneous cell problem is reached at the second order of $\varepsilon$. The full derivation of the homogenized equation, for Metropolis and Arrhenius microscopic dynamics and possible reaction terms, can be found in Appendix A. Here, the homogenized equation (leading order solution) for the purely diffusive cases is:

$$
\frac{\partial u_{0}}{\partial t}=\sum_{k} \frac{\partial}{\partial x_{k}}\left[\sum_{i} D_{k i}^{e f f} u_{0}\left(1-u_{0}\right) \frac{\partial}{\partial x_{i}}\left(\ln \left[\frac{u_{0}}{\left(1-u_{0}\right)}\right]-\beta a_{0} b_{0} J * u_{0}\right)\right]
$$

with: 


$$
\begin{array}{ll}
a_{0} & =\frac{1}{\Omega} \int_{Y} a(y) d y \\
b_{0} & =\frac{1}{\Omega} \int_{Y} b(y) d y \\
\Omega & =\int_{Y} d y \\
D_{k i}^{e f f}=\frac{1}{\Omega} \int_{Y}\left(D\left(\delta_{i k}+\frac{\partial \chi_{i}}{\partial y_{k}}\right)\right) d y & \text { for Metropolis dynamics } \\
D_{k i}^{e f f}=\frac{1}{\Omega} e^{-a_{0} b_{0}{ }^{*} u_{0}} \int_{Y}\left(D\left(\delta_{i k}+\frac{\partial \chi_{i}}{\partial y_{k}}\right)\right) d y & \text { for Arrhenius dynamics. }
\end{array}
$$

Here $\delta$ is the Kronecker symbol and $\chi$ is the solution of:

$$
\sum_{k} \sum_{l}\left[\frac{\partial}{\partial y_{l}}\left(D \frac{\partial \chi_{i}}{\partial y_{k}}\right)\right]=-\sum_{l} \frac{\partial D}{\partial y_{l}}
$$

over the heterogeneity scale $l_{h}$. All the summations extend over the dimensionality of the problem.

The above equations can be extended to include additional processes as well. For example, when a first order reaction is considered, Eq. 8 becomes:

$$
\frac{\partial u_{0}}{\partial t}=\sum_{k} \frac{\partial}{\partial x_{k}}\left[\sum_{i} D_{k i}^{e f f} u_{0}\left(1-u_{0}\right) \frac{\partial}{\partial x_{i}}\left(\ln \left[\frac{u_{0}}{\left(1-u_{0}\right)}\right]-\beta a_{0} b_{0} J * u_{0}\right)\right]-k_{r}^{e f f} u_{0}
$$

where

$$
k_{r}^{e f f}=\frac{1}{\Omega} \int_{Y} k_{r} d Y .
$$

We close this section with a brief discussion on the other scaling regimes. When the potential range is of the same order as the scale of the heterogeneity, only the definition of the potential $\mathrm{K}$ differs from the above case and becomes:

$$
\mathrm{K}\left(\mathrm{y}_{\mathrm{A}}, \mathrm{y}_{\mathrm{B}}\right)=\mathrm{Z}\left(\left|\mathrm{y}_{\mathrm{A}}-\mathrm{y}_{\mathrm{B}}\right|\right) \mathrm{a}\left(\mathrm{y}_{\mathrm{A}}\right) \mathrm{b}\left(\mathrm{y}_{\mathrm{B}}\right)
$$

Here both the distance term and the medium indicator term in the interaction potential depend only on the microscale. Because of the scaling within the distance term, the potential $\mathrm{Z}$ is of the form ${ }^{14}$ :

$$
\mathrm{Z}=\varepsilon^{-\mathrm{n} J}\left(\frac{\mathrm{x}_{\mathrm{A}}}{\varepsilon}-\frac{\mathrm{x}_{\mathrm{B}}}{\varepsilon}\right)=\varepsilon^{-\mathrm{n}} \mathrm{J}\left(\mathrm{y}_{\mathrm{A}}-\mathrm{y}_{\mathrm{B}}\right)
$$

where $\mathrm{n}$ is the dimensionality of the problem. Succinctly, this scaling arises from the fact that the macroscale system should perceive the contribution of particles only in a small region of the domain of the order of the potential range. The derivation of the effective mesoscopic equation in this case is not shown but is analogous to the previous case with the exception that the inhomogeneous cell problem is reached for terms of fourth order in $\varepsilon$, so the algebra becomes more complicated. Finally, for the remaining case, where the interaction range is much smaller than the heterogeneity, a second microscale variable must be introduced: 


$$
\eta=\frac{1_{\mathrm{p}}}{1_{\mathrm{h}}}<<1 \text { and } \mathrm{s}=\frac{\mathrm{y}}{\eta} .
$$

For example, if $\eta=\varepsilon^{\mathrm{m}}$, the distance term in the interaction potential could be written as:

$$
\mathrm{Z}=\varepsilon^{-\mathrm{nm}} \mathrm{J}\left(\frac{\mathrm{y}_{\mathrm{A}}}{\varepsilon^{\mathrm{m}}}-\frac{\mathrm{y}_{\mathrm{B}}}{\varepsilon^{\mathrm{m}}}\right)=\varepsilon^{-\mathrm{nm}} \mathrm{J}\left(\mathrm{s}_{\mathrm{A}}-\mathrm{s}_{\mathrm{B}}\right) .
$$

In the next sections, an overview of the numerical techniques used is given, followed by the presentation of the numerical validation of the effective mesoscopic equation for the first scaling regime. Finally, direct numerical results for various scaling regimes are presented to elucidate the role of heterogeneity in concentration patterns and effective permeation properties.

\section{NUMERICAL TECHNIQUES}

The mesoscopic equations presented are solved in two dimensions using spectral methods along with a Newton-Krylov solver ${ }^{32}$. The boundary conditions are chosen so that an overall gradient is present in the $\mathrm{x}_{1}$ direction, where a Chebyshev polynomials based approach is used. In the perpendicular $\mathrm{x}_{2}$ direction, periodic boundary conditions are used, and a fast Fourier transform (FFT) based approach is employed. The use of Chebyshev polynomials as interpolant requires an unequally spaced discretization defined by the Chebyshev points 33 :

$$
\mathrm{x}_{\text {cheb }}(\mathrm{k})=\cos \left(\frac{\pi \mathrm{k}}{\mathrm{n}}\right) \text { with } \mathrm{k}=1, \ldots, \mathrm{n} .
$$

Although Unequally-Spaced Fourier transform (USFFT) procedures do exist 34, 35, for simplicity, here the convolution products in the mesoscopic equations are computed on an interpolated uniform grid, along with virtual induced periodicity 20 in the direction of the gradient so that regular FFT can finally be used. The virtually periodic extended domain also accommodates the boundary conditions as shown in Figure 3.

For simplicity, a piecewise constant interaction potential J, used also in Figure 1, is employed:

$$
\begin{array}{ll}
J\left(\mid r-r^{\prime}\right)=\frac{w}{N_{p}} & \text { if }\left|r-r^{\prime}\right| \leq l_{p}, \\
J\left(\mid r-r^{\prime}\right)=0 & \text { if }\left|r-r^{\prime}\right|>l_{p},
\end{array}
$$

where $\mathrm{w}$ is the interaction strength, $\mathrm{l}_{\mathrm{p}}$ is the interaction range, and $\mathrm{N}_{\mathrm{p}}$ is the number of neighbors within the radius of the cutoff distance of the potential.

For the solution of the homogenized problem, given the possible variations of the diffusivity, an FFT approach with fully periodic boundary conditions is used to solve Eq. 14. Its solution permits the complete evaluation of the integrals in Eq. 12 and 13 by an adaptive recursive Simpson's rule scheme (MatLab). The effective diffusion coefficients given by Eq 12 and 13 can subsequently be used in Eq. 8. Being of the same form as the original mesocopic equations, the homogenized Eq. 8 is solved using the spectral techniques described above.

Numerically, the heterogeneity in properties is introduced using arctangent functions for smoothness and mathematical continuity at interfaces between different materials. An example of variable properties across the period of heterogeneity is depicted in Figures $4 \mathrm{c}$ and $4 \mathrm{~d}$. For simplicity, we focus on two materials and use the terminology of material and defects to indicate variation between two sets of properties. The whole procedure has been implemented in Matlab. For most simulations, 64x64 nodes are used. 


\section{NUMERICAL RESULTS}

First we assess the accuracy of the homogenized, effective mesoscopic equations derived above. Then, the effect of materials heterogeneity on concentration patterns and flux are examined for a simple configuration before studying the effect of defect dispersion.

\section{Validation of the homogenized equations}

To numerically assess the accuracy of the effective mesoscopic equation when the potential range is much larger than the heterogeneity scale, direct numerical simulations are performed for a system satisfying all the relevant assumptions. The simulated model system is depicted in Figure 4a, whereas Figure 4b shows a blowup where white areas correspond to defects an the shaded ones to the material. The diffusivity and potential of interactions across the period of heterogeneity are also depicted in Figures $4 \mathrm{c}$ and $4 \mathrm{~d}$, respectively.

The fluxes obtained from the effective mesoscopic equations and direct numerical simulations as a function of spatial location (in the direction perpendicular to the transport gradient) are shown in Figure 4e for two cases. In case (1), heterogeneity is considered in the interaction potential only, and the diffusion is constant (dashed line in Figure 4c). In case (2), the diffusion coefficient varies as well as shown in Figure 4c. The dashed horizontal lines depict the results from the homogenized equation, whereas the oscillatory profiles indicate the spatially varying flux at the outlet as obtained from direct numerical simulations. The corresponding spatially average fluxes of direct numerical simulations are shown as horizontal solid lines.

In both cases, the results compare well between the two approaches within the accuracy expected. Indeed, the effective mesoscopic equations are only the leading order equations to which correction terms (of order $\varepsilon$ or higher) can be added. The substantial spatial variation of flux in case 2 is a result of the strong variation in diffusivities between the normal material and the defects. Note that due to the homogenization procedure, the spatial information in both concentrations and fluxes cannot generally be reconstructed from the solution of the homogenized equation. However, if one is interested in average properties of the entire system, such as permeability, the homogenized equations suffice.

\section{Effect of heterogeneity on concentration patterns and flux profiles}

To elucidate the effect of heterogeneity on the system $\mathrm{s}$ behavior, we start with a simple heterogeneous configuration of two stripes (material and defect) running along the concentration gradient, as depicted in Figure 5a. Several variations in the diffusivity and interaction potential are introduced, including the limiting cases of simple Fickian diffusion (case 1) and pure material with interparticle interactions and no defects (case 2). In the other cases, the interaction potential is taken to be piecewise constant and attractive in the normal material, whereas particles do not interact in the defect. The material adjacent to the inlet and outlet, where concentrations of $\mathrm{u}=1$ and $\mathrm{u}=0$ are imposed, is taken to be of the same nature as the normal one. Microscopic Arrhenius dynamics is considered.

Figures $5 \mathrm{~b}$ and $5 \mathrm{c}$ show the isoconcentration contours and streamlines for case 3 where the diffusivities in the material and defect are equal. Overall, Fickian behavior within the defected domain, and Arrhenius-type behavior, in the normal material, are observed. The streamlines in panel $\mathrm{c}$ show that considerable mixing occurs in the direction normal to the imposed gradient. Furthermore, substantial uphill diffusion happens due to attractive interparticle interactions within the normal material. The variation in the diffusivity within the defect (cases 4 
and 5) significantly alters the mixing patterns and the flux at the outlet, as shown in Figure 5d. These results indicate that coupling between the material and the defect result in effective properties that are not merely geometrically weighted means of individual elements of the system.

\section{Effect of defect dispersion and membrane asymmetry}

In order to examine the influence of the dispersion of the heterogeneity on the concentration patterns and on the flux at the outlet, direct numerical simulations for different sizes of defects, with defects occupying a constant percentage $(25 \%)$ of the total area, were performed. In the square-shaped defects, no interparticle interactions occur and the diffusivity is low whereas in the normal material, particles attract each other according to a piecewise constant potential and diffuse with a constant diffusivity. Both Arrhenius and Metropolis microscopic dynamics were examined. The range of the interaction potential was varied as well in order to examine how solutions vary at different scaling regimes.

Figure $6 a$ shows a typical concentration pattern for $l_{\mathrm{p}}=5$ and $l_{\mathrm{h}}=16$ using Metropolis dynamics. Here again, considerable two-dimensional mixing in the transverse direction of the gradient is observed. The overall flux at the outlet as a function of the period of the heterogeneity is depicted in Figure $6 \mathrm{~b}$ for Arrhenius and Metropolis dynamics and different potential length scales. The results indicate that even for a given density of defects, their distribution can change the outlet flux by as much as twofold, especially in the Arrhenius case where larger gradients in concentration occur near the entrance of the membrane. This microstructure effect rationalizes one plausible mechanism responsible for the variation of permeabilities of different membranes. It can also be observed in Figure $6 \mathrm{~b}$ that the dependence of the permeability on the dispersion of defects is non-monotonic due to concentration patterns and 2D mixing. Although the parameters chosen here do not comply with the weak convergence assumption for the applicability of the homogenized equations, panel $\mathrm{b}$ shows that, for $l_{\mathrm{p}}=32$, they still give a reasonable estimate, when the heterogeneity scale to macroscale ratio is of the same order as the variations in amplitude of the potential, as happens when $l_{h} \sim 10$ (significant deviations are observed for $l_{h}=4$ and $l_{h}=32$ ).

Finally, simulations have been performed to examine the role of the position of defects in the overall permeation. Figure 7 summarizes results from various configurations for two different heterogeneity (periodicity) scales as indicated. In panel a, various fractions of defects are introduced near the low-pressure side, whereas in panel b, they are introduced near the highpressure side. Comparison between the two panels indicates an asymmetry in operation mode, due to the position of defects, as illustrated by differences of up to a factor of two in fluxes, for the same fraction of defects. In particular, it appears that defects are much more important in regions close to the inlet (high-pressure side), where the concentrations are high and interaction forces contribute largely to the local behavior. In high concentration regimes, the scale of the heterogeneity also plays an important role as seen in panel $b$.

Recent laser confocal optical microscopy studies of the microstructure of zeolite membranes indicate that membranes fabricated using the secondary growth technique, i.e., formed from deposition of an initial coating of particles on a substrate, have larger grain boundaries near the zeolite/substrate interface ${ }^{21}$. As a result, one may expect an asymmetry in fluxes in the case of strong intermolecular forces, depending on which side is exposed to the high-pressure stream. 


\section{CONCLUSIONS}

A new mathematical framework linking molecular scale information with macroscopic scale (effective) properties of heterogeneous materials was introduced. Heterogeneity in materials is the rule rather than the exception in practice. The approach presented here was applied to the permeation of two-dimensional model membranes. The proposed approach replaces molecular simulations with a mesoscopic approach that enables us to reach longer time and length scales. Furthermore, due to its continuum nature, mesoscopic models render the inclusion of heterogeneity over various scales tractable through the use of homogenization techniques. Direct numerical simulations were also carried out giving good agreement with the results obtained using homogenization. The influence of the nature of the heterogeneity (potential and diffusivity) and the effect of defect density and dispersion were also examined to delineate qualitatively the relationship between material structure and effective properties.

We have found that, as expected, the introduction of heterogeneity significantly affects the permeation properties. Furthermore, heterogeneity can also make evident uphill diffusion. Although, defect density plays a significant role in the permeation properties, defect dispersion is also important giving rise to asymmetric permeation behavior when intermolecular forces are significant. Finally, although physical conclusions were drawn, our focus has been on proof-ofthe-concept, namely, the applicability of homogenization techniques, widely used for modeling of mechanical properties of composite materials, to systems with interparticle interactions. This framework offers a promising approach for modeling complex systems with heterogeneities, such as polycrystalline membranes, in which molecular scale information dictates macroscopic properties. Future work will focus on practical systems.

\section{ACKNOWLEDGMENTS}

This research was partially supported by National Science Foundation through CTS-9702615, CTS-9904242, DMS-9626904, and DMS-9801769. 


\section{REFERENCES}

1 N. Metropolis, A.W. Rosenbluth, M.N. Rosenbluth, A.H. Teller, and E. Teller, Journal of Chemical Physics 21, 1087 (1953).

2 K. Binder, Advanced Materials 4, 540 (1992).

3 K. Binder, (Springer-Verlag, Berlin, 1986), Vol. 7.

4 M.P. Allen and D.J. Tildesley, Computer simulation of liquids (Oxford Science Publications, Oxford, 1989).

5 T.B. Thompson, DOE workshop roadmap for computational chemistry , http://itri.loyola.edu/molmodel (1999).

6 P.T. Cummings, B.K. Peterson, C.K. Hall, M. Neurock, A.Z. Panagiotopoulos, and P.R. Westmoreland, NSF Workshop report, http://flory.engr.utk.edu/nsf (1997).

7 S.M. Auerbach, Int. Rev. Phys. Chem. 19, 155 (2000).

8 H. Chuan Kang and W.H. Winberg, Chem. Rev. 95, 667 (1995).

9 G. Gilmer, Science 208, 355 (1980).

10 R. Gomer, Report and Progress in Physics 53, 917 (1990).

11 V.P. Zhdanov and B. Kasemo, Surface Science Reports 20, 111 (1994).

12 R.M. Ziff, E. Gulari, and Y. Barshad, Physical Review Letters 56, 2553 (1986).

13 A. Turing, Phil. Trans. R. Soc. London Ser. B 237, 37 (1952).

14 D.J. Horntrop, M.A. Katsoulakis, and D.G. Vlachos, Journal of Computational Physics , submitted (2000).

15 G. Giacomin and J.L. Lebowitz, SIAM J. Appl. Math. 58, 1707 (1998).

16 M. Hildebrand and A.S. Mikhailov, Journal of Physical Chemistry 100, 19089 (1996).

17 D.G. Vlachos and M.A. Katsoulakis, Physical Review Letters 85, 3898 (2000).

18 A. De Masi, E. Orlandi, E. Presutti, and L. Triolo, Proc. Royal Soc. Edinb 124A, 1013 (1994). 
19 M.A. Katsoulakis and D.G. Vlachos, in Mesoscopic modeling of surface processes (Institute of Mathematics and its Applications, Springer-Verlag, 2000), to appear.

20 R. Lam, D.G. Vlachos, and M.A. Katsoulakis, in progress (2001).

21 G. Bonilla, M. Tsapatsis, D.G. Vlachos, and G. Xomeritakis, J. Membrane Sci. 182, 103 (2000).

22 E. Sanchez-Palencia, Non-homogeneous media and vibration theory (Springer-Verlag, Berlin, New York, 1980).

23 E. Sanchez-Palencia and A. Zaoui, Homogenization techniques for composite media: lectures delivered at the CISM International Center for Mechanical Sciences, Udine, Italy, July 1-5, 1985 (Springer-Verlag, Berlin, New York, 1986).

24 A. Bensoussan, J.-L. Lions, and G. Papanicolaou, Asymptotic analysis for periodic structures (North-Holland, Amsterdam, New York, 1978).

25 M.E. Cruz and A.T. Patera, International Journal for Numerical Methods in Engineering 38, 1087 (1995).

26 A. Bouddour, J.-L. Auriault, and M. Mhamdi-Alaqui, International Journal of Heat and Mass Transfer 41, 2263 (1998).

27 C.C. Mei, J.-L. Auriault, and C.-o. Ng, in Advances in applied mechanics, edited by J. W. Hutchinson and T. Y. Wu (Academic Press, San Diego, 1996), Vol. 32, p. 277.

28 J.-L. Auriault and H.I. Ene, International Journal of Heat and Mass Transfer 37, 2885 (1994).

29 D. Cioranescu and J. Saint Jean Paulin, Homogenization of reticulated structures (Springer-Verlag, New York, 1998).

30 M.A. Murad, International Journal for Numerical and Analytical Methods in Geomechanics 23, 673 (1999).

31 J. Poutet, D. Manzoni, F. Hage-Chehade, C.J. Jacquin, M.J. Bouteca, J.-F. Thovert, and P.M. Adler, Journal of the mechanics and physics of solids 44, 1587 (1996).

32 C.T. Kelley, Iterative Methods for Linear and Nonlinear Equations: Matlab Codes, 1995.

33 L.N. Trefethen, Spectral methods in MATLAB (Society for Industrial and Applied Mathematics (SIAM), Philadelphia, PA, 2000).

34 G. Beylkin, Appl. Comput. Harmon. Anal 2, 363 (1995). 
35 W.H. Press and G.B. Rybicki, Astrophysical Journal 338, 227 (1989). 


\section{FIGURE CAPTIONS}

Figure 1: Comparison of 1D (a) and 2D (b) concentration profiles from gradient continuous time MC (solid lines) and mesoscopic simulations (dashed lines) for various lengths of the intermolecular potential indicated. The inset in panel a depicts the geometry studied in 1D along with the boundary conditions. The potential in these simulations is $\beta \mathrm{KN}_{\mathrm{p}}=4$ up to a distance of $1_{\mathrm{p}}$ and zero otherwise.

Figure 2: Schematic of the problem showing the macroscale $\mathrm{x}(\mathrm{a})$, the microscale $\mathrm{y}(\mathrm{b})$, the potential (c), and the typical microstructure obtained from fluorescence laser confocal microscopy of a supported MFI zeolite membrane (from ${ }^{21}$ ).

Figure 3: Schematic of the induced virtual periodicity for application of FFT in the direction of transport.

Figure 4: Schematic of the system configuration (a), magnification of heterogeneous medium with normal material (shaded) and defects (white squares), diffusion coefficient (c) and interparticle potential (d) plotted in 1D, and fluxes at the outlet (e), from the homogenized equation (horizontal dashed lines) and direct numerical simulations (solid, oscillating lines). The solid horizontal lines are spatially averaged fluxes from direct numerical simulations. For these simulations, the heterogeneity in case (1) is in the potential $\left(\mathrm{N}_{\mathrm{p}} \beta \mathrm{w}_{\text {defect }}=3.6, \mathrm{~N}_{\mathrm{p}} \beta \mathrm{w}_{\text {material }}=4\right)$ and in case (2) in both the potential $\left(\mathrm{N}_{\mathrm{p}} \beta \mathrm{w}_{\text {defect }}=3.6, \mathrm{~N}_{\mathrm{p}} \beta \mathrm{w}_{\text {material }}=4\right)$ and the diffusion coefficient $\left(D_{\text {defect }} \sim 0, D_{\text {material }}=1\right)$. The parameters are $l_{p}=30$ and $l_{h}=4$. The homogenized equations are in good agreement with direct numerical simulations.

Figure 5: Configuration examined (a), iso-concentration contours for case (3) (b), streamlines for case (3) (c), and fluxes at the outlet (d) for different cases. Case (1): $D_{\text {defect }}=D_{\text {material }}=1$ and no interactions (only normal material with Fickian diffusion); case (2) $\mathrm{D}_{\text {defect }}=\mathrm{D}_{\text {material }}=1$ and same interparticle interactions everywhere (only normal material). In cases 3-5, interactions of strength $\mathrm{N}_{\mathrm{p}} \beta \mathrm{w}=4$ occur only in the material. Case (3): $\mathrm{D}_{\text {defect }}=\mathrm{D}_{\text {material }}=1$, case (4): $\mathrm{D}_{\text {defect }} \sim 0, \mathrm{D}_{\text {material }}=1$, case (5): $D_{\text {defect }}=2, D_{\text {material }}=1$. The parameters are $l_{p}=5$ and $l_{h}=64$. Uphill diffusion in the transverse direction is made apparent when defects are present.

Figure 6: Concentration pattern for $l_{p}=5$ and heterogeneities of period $l_{h}=16$ using Metropolis dynamics (a) and average fluxes at the outlet versus period of the heterogeneity (b). The parameters are $\mathrm{N}_{\mathrm{p}} \beta \mathrm{w}=4, \mathrm{D}_{\text {defect }} \sim 0$, and $\mathrm{D}_{\text {material }}=1$. The homogenized equations were also solved for $1_{p}=32$ (dashed line) although the assumption of weak convergence is not verified for the parameter chosen here

Figure 7: Effect of defect position for two different sizes of heterogeneity $\left(l_{h}=8\right.$ and $\left.l_{h}=16\right)$ and for various fractions of defects. Defect placed near the low-pressure side (a) and defect placed near the high-pressure side (b). The parameters are $D_{\text {defect }} \sim 0, D_{\text {material }}=1$, and $\mathrm{N}_{\mathrm{p}} \beta \mathrm{w}=4$. 